\title{
An Optical Calibration Manipulator System
}

Pierre-Andre Amaudruz, Frank Berghaus, Shaomin Chen, Richard Helmer, Corrie Holmberg, Keith Hoyle, Issei Kato, Peter Kitching, Akira Konaka, Mark Lenckowski, David Morris, Scott Oser

\begin{abstract}
The K2K experiment at KEK is searching for neutrino oscillations by measuring the neutrino beam flux at both a $1 \mathrm{kt}$ water Cherenkov detector (front detector) close to the neutrino source, and at the Super Kamiokande detector $250 \mathrm{~km}$ away. Part of the optical calibration of the front detector is accomplished by transmitting laser light via a fibre optic cable to a light diffusing ball immersed in the water. A manipulator system has been built to position the ball at various locations within the water volume. A description of the mechanics and the control system is presented.
\end{abstract}

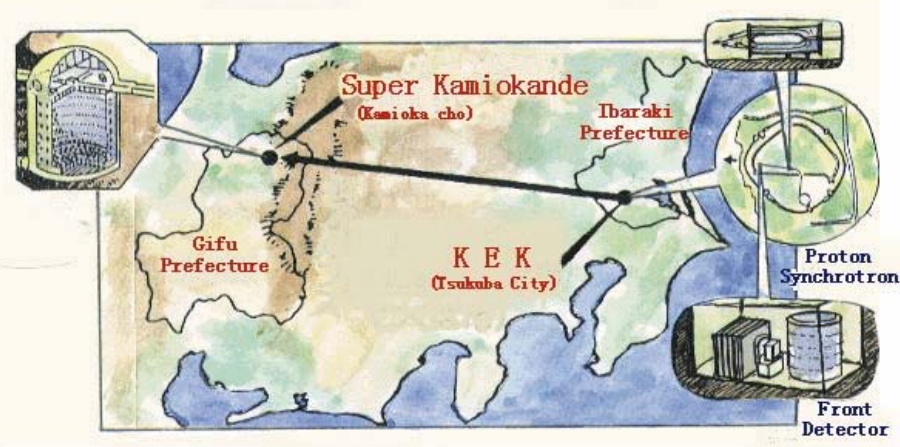

Figure 1 Route of neutrinos across Japan from KEK to Super Kamiokande

\section{INTRODUCTION}

The $\mathrm{K} 2 \mathrm{~K}$ experiment uses an accelerator-produced muon neutrino beam directed at the Super Kamiokande detector for the study of neutrino oscillations (see Fig. 1). The K2K front detector is a vertically oriented cylinder, located close to the neutrino source, ten metres across and ten metres deep containing $1 \mathrm{kt}$ of ultrapure water. Cherenkov light emitted by various interactions in the water is detected by 680 inward facing photomultiplier tubes (PMTs) viewing the detector volume. The purpose of the optical calibration is to correct the detector response for effects such as light attenuation in the water, PMT angular response, time delays introduced by varying transit time through the PMTs and associated cabling, and time walk of the signal with pulse amplitude.

This work is supported under grants from the British Columbia Knowledge Development Fund, the Canadian Foundation for Innovation and the Natural Sciences and Engineering Research Council of Canada.

Pierre-Andre Amaudruz, Shaomin Chen, Richard Helmer, Corrie Holmberg, Keith Hoyle, Issei Kato, Peter Kitching, Akira Konaka and David Morris are with TRIUMF, Vancouver, B.C., Canada

Mark Lenckowski is with TRIUMF, University of Victoria, Victoria, B.C., Canada

Frank Berghaus and Scott Oser are with the Department of Physics and Astronomy, University of British Columbia, Vancouver, B.C., Canada

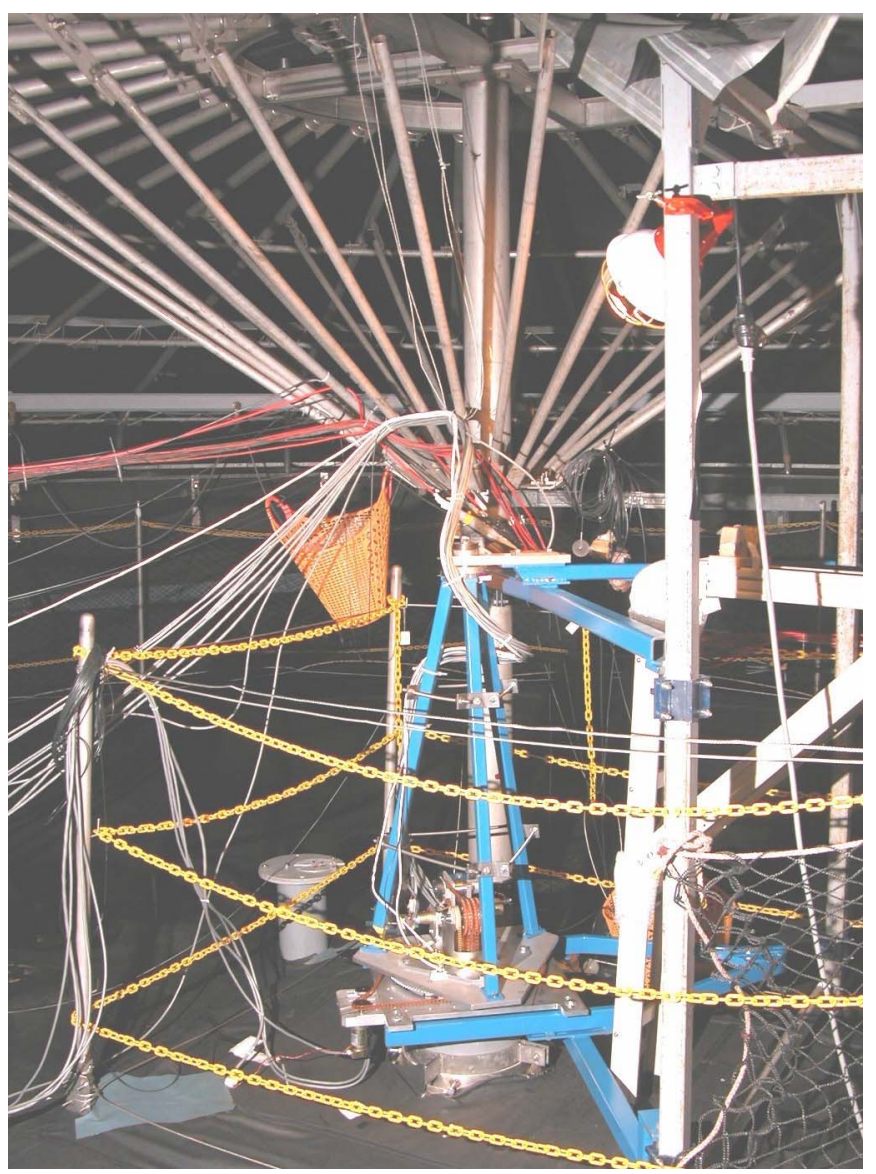

Figure 2 Installation on Detector Lid under Roof. The blue support bars are clamped to the existing platform frame. The three vertical blue bars make a support tripod, with a load bearing at the top, and the turntable at the bottom. A motor drives a large toothed gear attached to the bottom of the turntable via an orange composite drive chain. The motors for driving the manipulator arms are on the far side of the turntable, and drive the arms through a set of orange chains visible in the center of the turntable. The umbrella structure is the support for the roof of the detector.

The manipulator was designed to enable these variables to be probed throughout the detector volume. Installation was done in late April 2005, followed by one month of data collection, and removal in early June 2005.

\section{MECHANICAL DESIGN}

The manipulator consists of a 6 metre vertical column suspended in the detector from a platform mounted on the detector cover (see Fig. 2), with a three-jointed articulating arm (A, B, C segments) mounted from the bottom of the column (V segment). A light diffusing ball is mounted at the outer end of the third (C) segment. Four motors are used to rotate the column and drive the arm segments so as to position the ball in the desired location. 


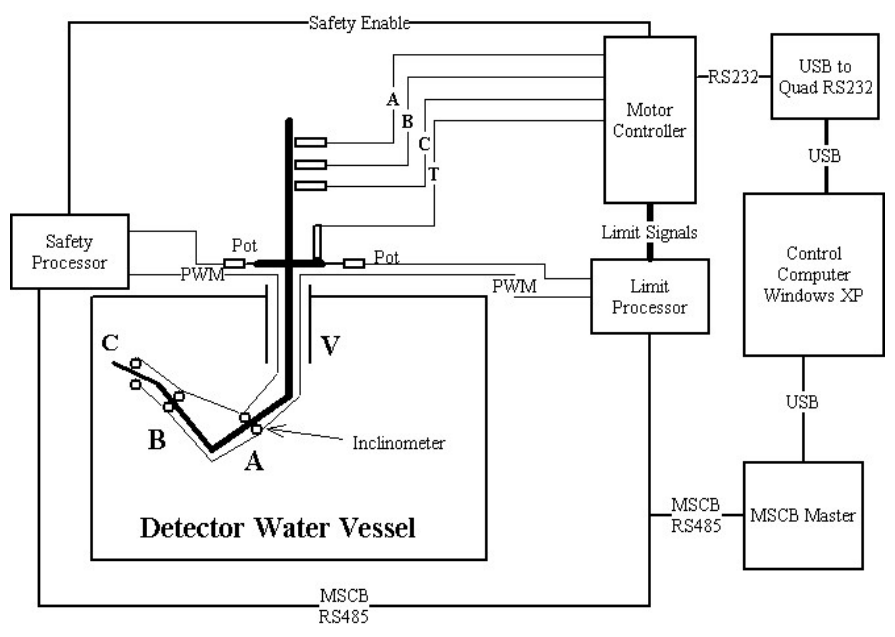

Figure 3 Block Diagram of Manipulator System

The drive for each arm segment is transmitted from the top of the vertical column via sprockets and drive chains. Each joint of the arm conveys the drive to the next arm segment, until the final arm segment is reached.

The types of materials that can be used for the system are limited so as to prevent contamination of the ultra-pure deionized water in the detector. Only stainless steel and some plastics are used.

The articulating arm segments are designed for neutral buoyancy in the water so as to minimize the forces required to move them. This was accomplished by sealing the ends of each segment.

\section{CONTROL SyStem DESIGN}

It is of paramount importance to be able to position the ball accurately and to ensure that no arm segment will contact any of the PMTs. The control system performs this using redundant position sensors and feedback systems to independent computers.

\section{A. Position Feedback}

The critical component in this system is the position feedback of each arm segment. Several feedback mechanisms, including limit switches, proximity sensors and potentiometers were considered but dismissed for various reasons - limit switches would rely too much on trusting the motor controller to maintain the current arm positions, potentiometers at each joint in the arm would lead to cumulative errors in the positions. As well, the only potentiometers found were oil filled and an oil leak would lead to unacceptable contamination of the water.

The feedback system adopted was based on solid state accelerometers using Micro-Electro-Mechanical System (MEMS) technology. Sensors using a thermal mass detection system [1] were chosen for their low noise characteristics. A small proof mass of gas is heated, creating a thermal gradient in the gas. During acceleration, the package moves, but the gas stays still. A set of thermal sensors measures the resulting change in temperature to determine the acceleration. In the present application, gravity provides the constant acceleration.

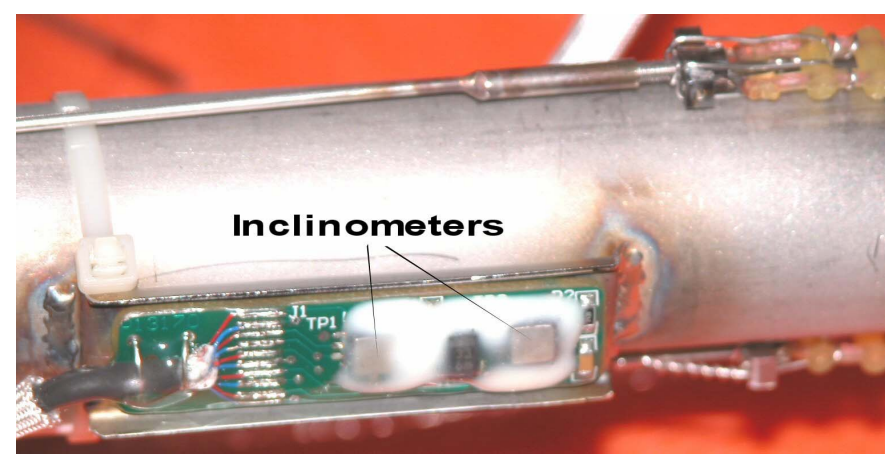

Figure 4 Inclinometer assembly mounted on arm

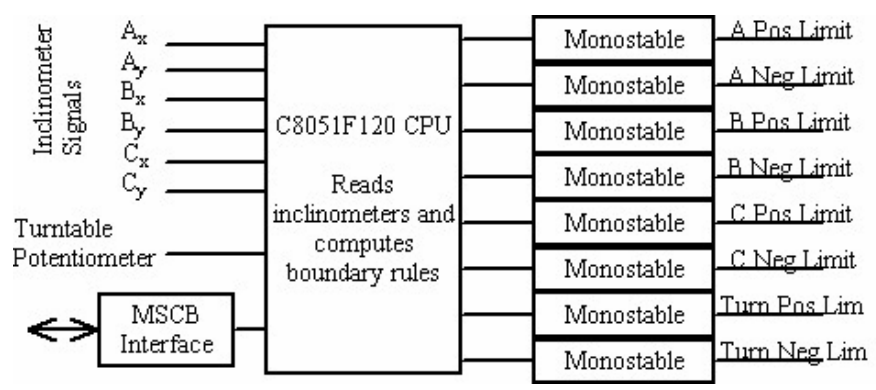

Figure 5 Inclinometer Processor Block Diagram

The units have two internal accelerometers at right angles to each other. The accelerometer resolution should be less than 1 milli-gravity, which translates into 0.09 degrees of motion. The output signal is a $100 \mathrm{~Hz}$ Pulse Width Modulated (PWM) signal.

The accelerometers (or inclinometers, as they are referred to in the present application since they are used to provide angular information) are mounted in pairs on small circuit boards. These are then wired using a small gauge flexible wire, encapsulated with a polyurethane coating and glued with epoxy to a mounting plate on each arm segment (see Fig. 4).

A total of six inclinometer assemblies are installed on the manipulator. The vertical column has two inclinometer assemblies mounted at 90 degrees to measure deflection of the column in two planes. Each movable arm segment has an inclinometer assembly mounted vertically. The B arm segment has an extra inclinometer mounted horizontally to measure vibration in the manipulator.

\section{B. Position Processing}

The inclinometer data is read and processed by two 8051 based processors, Limit and Safety [2]. The PWM signal is converted into angles for each arm and boundary conditions are then internally calculated to determine if there are violations that require motion limiting. Each processor reads one inclinometer from each inclinometer assembly to allow for redundancy. All outputs from the processor boards use watchdog circuits to improve reliability (see Fig 5). 
TABLE 1

RULE SET FOR LIMIT AND SAFETY PROCESSORS

\begin{tabular}{|l|l|}
\hline Rule & Protects from... \\
\hline Roof & Collision of B or C tips with roof \\
\hline Floor & Collision of B or C tips with floor \\
\hline Centre & Collision of B or C tips with vertical section \\
\hline Radial & Collision of B or C tips with outer radius \\
\hline VA & Minimum angle Vertical to A Arm joint \\
\hline AB & Minimum angle A Arm to B Arm joint \\
\hline BC & Minimum angle B Arm to C Arm joint \\
\hline Turntable & Over-rotation beyond $+/-185$ degrees \\
\hline
\end{tabular}

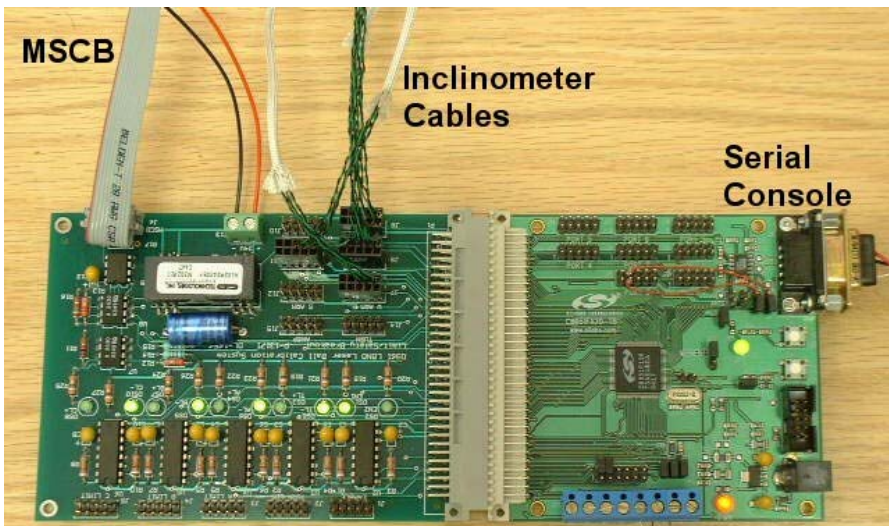

Limit Outputs

Figure 6 Limit Processor Board

\begin{tabular}{|c|c|c|c|c|c|c|}
\hline \multicolumn{6}{|c|}{ LaserBall Limit Processor - Build Apr 012005 14:55:22 } & \multirow{2}{*}{$\begin{array}{r}30198879 \\
37591 \\
0 \\
0 \\
0 \\
0 \\
0 \\
0\end{array}$} \\
\hline $\begin{array}{l}\text { A Arm } \\
\text { B Arm } \\
\text { C Arm } \\
\text { VArm } \\
\text { Arrm } \\
\text { A Arm }\end{array}$ & $\begin{array}{l}\times \text { Aug } \\
9300 \\
1450 \\
1450 \\
28800 \\
28750\end{array}$ & $\begin{array}{rr}\text { yfuvg perAug } \\
10200 & 19000 \\
2020 & 3456 \\
2020 & 3456 \\
25500 & 50700 \\
25550 & 50700\end{array}$ & $\begin{array}{l}\text { Offset } \\
-1.95 \\
-0.90 \\
1.37 \\
0.00 \\
0.00\end{array}$ & 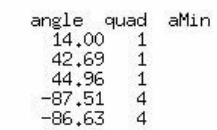 & 更 & \\
\hline $\begin{array}{l}\text { A Arm } \\
\text { B Arm } \\
\text { C Arm } \\
\text { V Arm } \\
\text { Verm }\end{array}$ & $\begin{array}{l}\text { Length } \\
2.100 \\
1.900 \\
0.123 \\
5.000 \\
5.000\end{array}$ & 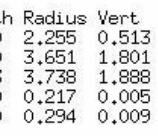 & \multicolumn{2}{|c|}{$\begin{array}{l}\operatorname{Lim}-\operatorname{Lim}+ \\
0 \times 100 \times 00 \\
0 \times 100 \times 00 \\
0 \times 100 \times 00\end{array}$} & & \\
\hline $\begin{array}{l}\text { Radial } \\
\text { Radial } \\
\text { Radial } \\
\text { Radial }\end{array}$ & $\begin{array}{l}\text { Offset } \\
\text { Safe } \\
\text { Max Rt } \\
\text { Max Lt }\end{array}$ & $\begin{array}{l}0.990 \\
3.400 \\
2.410 \\
4.390\end{array}$ & & $\begin{array}{l}\text { Turntable Raw } \\
\text { Turntable Pot } \\
\text { Turntable Angle } \\
\text { Turntable Lim + }\end{array}$ & $\begin{array}{l}2051 \\
4090 \\
0.557 \\
0 \times 00\end{array}$ & \\
\hline $\begin{array}{ll}\text { Vibr } X \\
\text { Vibr Y }\end{array}$ & & $\begin{array}{l}287503400.0 \\
25550200.0 \\
-14836\end{array}$ & & & & \\
\hline
\end{tabular}

Figure 7 Limit Processor Console

The Limit Processor (see Fig. 6) generates hardware limit signals for the four motors, which are connected to the Motor Controller, based on the boundary rules (see Table 1). These signals will prevent a motor's operation if it would carry that motor's arm further into a limit boundary that has been violated. The Safety Processor generates an enable signal which will disable the Motor Controller if a Safety boundary violation is detected. The boundaries for the Safety Processor define a volume slightly larger than the Limit Processor volume. Both processors have a console output to provide diagnostic information during operation (see Fig. 7).

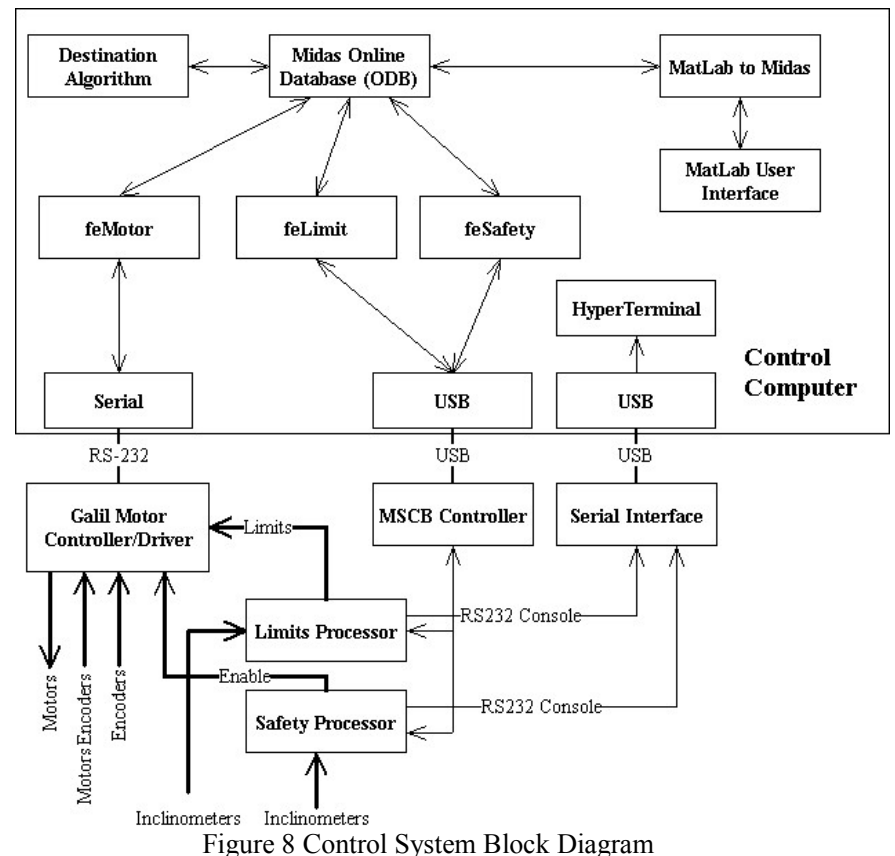

Figure 8 Control System Block Diagram

C. Motor Control

The four brushed DC gear motors are controlled by a four channel motor controller and driver module [3]. This controller uses an RS-232 connection for communication with the Control Computer. All motors have optical incremental encoders for speed and position feedback to the motor controller, and all axes have an auxiliary optical encoder mounted after the motor gearbox for finer position feedback. The auxiliary encoders have index marks which are connected to the controller index inputs to use as a homing reference in the event of a loss of position in the motor controller.

\section{Data Acquisition and Arm Control}

The Maximum Integration Data Acquisition System (MIDAS), developed at PSI [4], is used to implement an online database (ODB). Three MIDAS front end programs (feMotor, feLimit, feSafety) link the ODB to the motion controller and inclinometer processors. The Destination Algorithm program acts as an interface between the user's motion request, calculates destination values for the motors, defines complex motion profiles for linear motion of the probe tip, and implements a sequencer for moving the motors in a coordinated fashion (see Fig. 8).

The three front end programs and the Destination Algorithm program use a callback mechanism of the ODB to trigger functions on a change of value of a database entry. This results in the update of a constant parameter such as a boundary value in the case of feLimit and feSafety, a motion command to start a move to a new destination for feMotor, or a request to build a new complex table of motion coordinates in the case of the Destination Algorithm program.

The MIDAS logger can be run to collect and log all data associated with the front ends, to allow reconstruction of particular movements of the probe. 


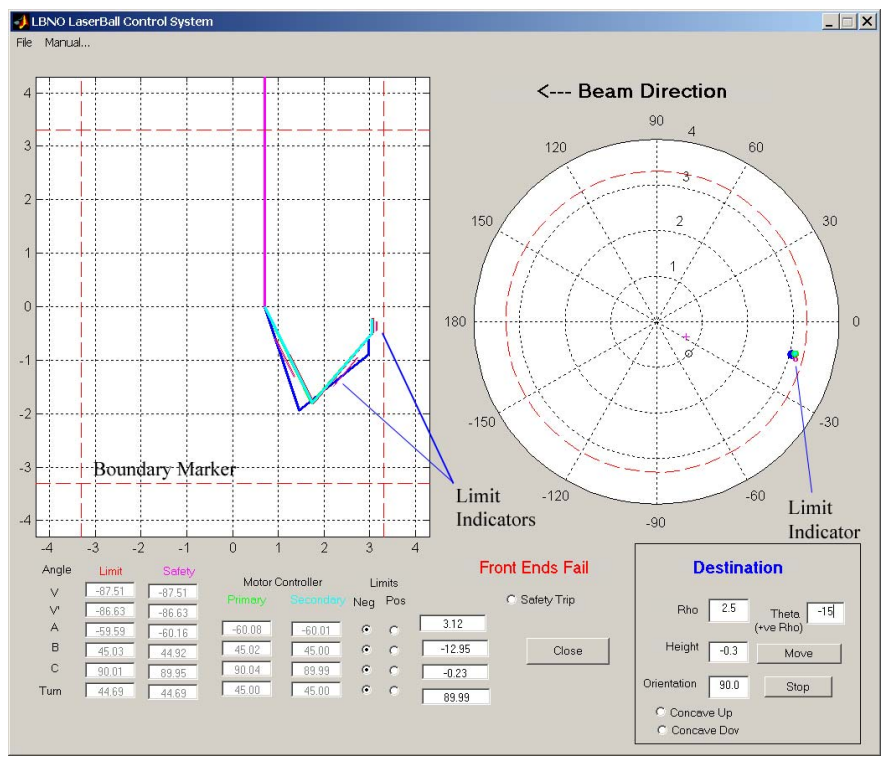

Figure 9 User Interface

\section{E. $M S C B$}

The inclinometer processors are running an application based on the MIDAS Slow Control Bus (MSCB), originally developed for power supply control. The MSCB was chosen to take advantage of a clean interface to MIDAS and speed the design process. An existing framework allows the developer to add application specific code and hardware using an 8051 based microprocessor. A standard development board for the processor was purchased, and a hardware specific card containing the MSCB interface, inclinometer inputs and limit signal outputs was built (see Fig. 6).

\section{F. User Interface}

MatLab was used to build the graphical user interface for the system (see Fig. 9 and Fig. 10). The interface displays both profile and polar views of the manipulator arm to assist the operator in visualizing arm movement and for diagnosing problems that are preventing motion. An interface layer was written to translate MatLab calls into MIDAS ODB calls. Motion requests are placed into the ODB, the Destination Algorithm program processes the request and sends the results back to the ODB, and the feMotor program sends the commands to the motor controller.

In the profile view each movable arm segment shows the limit status for its associated motor with separate fine lines next to the line depicting the arm segment. In the polar view, small dots on each side of the larger dot representing the tip of the probe show limits for the turntable.

\section{PERFORMANCE ISSUES}

\section{A. Inclinometers}

The inclinometers were very successful as a tool for this unique application. Non-cumulative errors between arm segments, light weight, no static friction and good angular resolution produced excellent position information. The

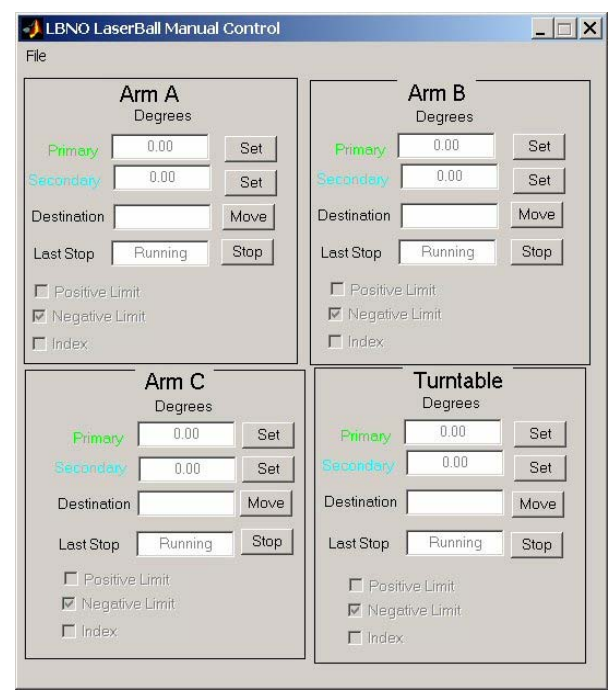

Figure 10 Manual Axis Interface
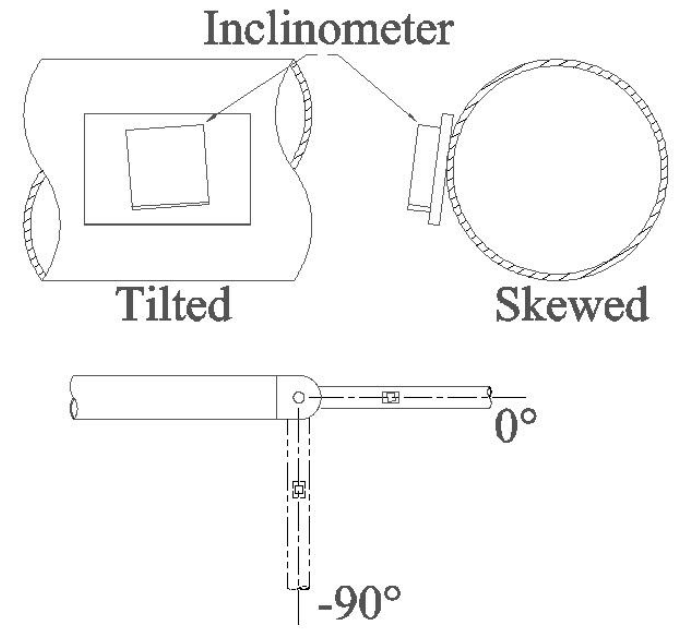

Figure 11 Inclinometer mounting

alignment when mounting is critical for good operation. A tilted inclinometer is easily corrected with an angular offset. A skewed inclinometer leads to position errors that must be corrected offline (see Fig. 11).

Three inclinometers failed during the calibration run in the detector. Both inclinometers on one of the assemblies mounted on the vertical section and the safety inclinometer on the $\mathrm{C}$ arm stopped operating. The failures did not compromise the operation of the manipulator. The vertical section inclinometers are not critical components for the manipulator operation. The limit inclinometer on the $\mathrm{C}$ arm was connected to the Safety processor to allow continued operation. These failures may be the result of water permeating the coating on the inclinometer assemblies.

\section{B. MIDAS}

Using MIDAS software led to a modular design with an effective common database for interprocess communication. 
Using the MSCB interface to read out the inclinometer data simplified the design. An existing framework allowed fast development of the Limit and Safety processor boards. Problems can arise when the MSCB requests data from the boards. During readout of the inclinometers the processor cannot be interrupted, but the MSCB does not tolerate delays in its protocol. This was resolved by discarding a reading that is interrupted by an MSCB request.

Some options to avoid this problem could include:

1) A TCP/IP based interface that can communicate with the ODB directly (under development at PSI). The Limit and Safety processors could then initiate updates to the ODB themselves. This would also remove two front end programs from the Control Computer.

2) Replace the software read of each inclinometer with a hardware solution. Use a gate array to build a set of counters for the inclinometers, with internal data latches and a bus interface to connect to the 8051 processor. This offloads the reading from the inclinometer processor, and prevents the MSCB from interfering with the data collection. A second 8051 processor and a dual port memory could also be used to implement this solution.

\section{CONCLUSIONS}

The system operated well during the calibration run in the detector. The inclinometers were the right device to give good angular position data without the problems associated with mechanical devices. Redundancy of the inclinometers and processor boards ensured that a safe operating environment could be maintained even with some component failures. The motor controller implemented several sophisticated algorithms for precise position control, and integrated well into the control system. The mechanical components functioned as expected .

\section{ACKNOWLEDGEMENT}

The authors wish to thank the TRIUMF Electronics Services Group for their assistance in designing and assembling components, and the Beamlines Group for calibration measurements.

\section{REFERENCES}

[1] MEMSIC Inc. MXD2020GL www.memsic.com

[2] Silicon Laboratories C8051F120 www.silabs.com

[3] Galil DMC-2142 and AMP-20440 www.galilmc.com

[4] Paul Scherrer Institute MIDAS midas.triumf.ca 